\title{
El cotidiano del cuidador familiar del adulto mayor con dependencia: reconfigurando el espacio
}

\author{
Virginia Reyes-Audiffred,* Zoila Esperanza Leitón-Espinoza**
}

\begin{abstract}
RESUMEN
Introducción: El cuidador familiar de un adulto mayor dependiente, al brindar cuidados durante las 24 horas del día, se enfrenta a situaciones complejas que pueden deteriorar su salud. Objetivos: Describir, analizar e interpretar el cotidiano del cuidador familiar del adulto mayor con dependencia. Metodología: Es una investigación de abordaje cualitativo; se realizó con ocho cuidadores familiares de adultos mayores con dependencia. La información se obtuvo a través de entrevistas a profundidad con un cuestionario de preguntas semiestructuradas. Se realizó análisis temático según Minayo, a partir del que se construyeron las categorías y subcategorías de las unidades temáticas. Resultados: Emergieron dos categorías: "tiempo y espacio síntesis de lo vivido", y "simbolizando el cuidado". En el presente artículo se describe la subcategoría "reconfigurando el espacio" de la categoría "tiempo y espacio síntesis de lo vivido". Conclusiones: Los discursos reflejaron que el cuidador familiar en su cotidiano se enfrenta a diversas situaciones, como compartir el espacio con el adulto mayor, así como introducir diferentes objetos como equipo médico y ayudas técnicas para poder brindar un cuidado seguro.
\end{abstract}

Palabras clave: Cotidiano, cuidador familiar, adulto mayor dependiente.

\section{Everyday family caregiver of older adults with dependence: reconfiguring space}

\begin{abstract}
Introduction: A family caregiver of a dependent elder providing care for 24 hours a day faces complex situations that can damage his/her health. Objective: To describe, analyze and interpret the day-today of a family caregiver of a dependent elder. Methodology: A qualitative research approach was conducted with eight family caregivers of older adults with dependence. The information was obtained through in-depth interviews with a questionnaire of semi-structured questions. Thematic analysis was performed according to Minayo, from which the categories and sub-categories of thematic units were built. Results: Two categories emerged: "time and space synthesis of lived experience", and "symbolizing care". The

\footnotetext{
* M.C.E. Profesora de Carrera Asociada C, tiempo completo. Escuela Nacional de Enfermería y Obstetricia de la Universidad Nacional Autónoma de México. Alumna del Programa de Doctorado en Ciencias de la Enfermería, Convenio Universidad Nacional de Trujillo-Universidad Nacional Autónoma de México, México. Parte de la tesis de Doctorado en Ciencias de Enfermería "El cotidiano del cuidador familiar del adulto mayor con dependencia”. Universidad Nacional de Trujillo, Perú-Universidad Nacional Autónoma de México, México.

** Doctora en Salud Pública. Docente de tiempo completo de la Facultad de Enfermería. Universidad Nacional de Trujillo, Perú. Convenio
} UNAM-UNT.
\end{abstract}

Recibido para publicación: 08/05/2014. Aceptado: 22/05/2014.

Correspondencia: Mtra. Virginia Reyes Audiffred. Camino Viejo a Xochimilco y Viaducto Tlalpan s/n, Col. San Lorenzo Huipulco, 14370, Delegación Tlalpan, México, D.F.

Tel: 56553181, ext. 206 y 207. E-mail: virginiar66@yahoo.com

Este artículo puede ser consultado en versión completa en http://www.medigraphic.com/enfermerianeurologica 
present article describes the subcategory "reconfiguring space" of the category "time and space synthesis of lived experience". Conclusions: The speeches reflected that the family caregiver faces various situations in his/her day-to day, such as sharing space with the elder, as well as introducing different objects such as medical equipment and assistive devices to provide safe care.

Key words: Daily, family caregivers, dependent elderly.

\section{INTRODUCCIÓN}

$\mathrm{S}$ egún datos del Instituto Nacional de Estadística y Geografía, las personas mayores de 60 años representan el $9.1 \%$ de la población total, ${ }^{1}$ y se proyecta que cada año aumente de forma paralela al incremento de la esperanza de vida, de tal forma que se espera que para el 2030 representen el 12\%. ${ }^{2}$ Es a partir de los 60 años de edad que se acentúa el proceso de envejecimiento, por lo que se hacen evidentes cambios biológicos, psicológicos y sociales que dan como resultado deficiencias funcionales y aumento de la vulnerabilidad ante situaciones de enfermedad. ${ }^{3}$ Aunado a lo anterior, en este grupo de edad se presentan complicaciones de enfermedades previas, como la enfermedad cerebro vascular (EVC), ${ }^{2}$ en la cual el organismo se ve afectado en diversas áreas, desde lo físico hasta lo cognitivo. Entre los daños motrices, se presentan deficiencias y discapacidades para caminar, manipular objetos y coordinar movimientos, así como para utilizar el brazo y la mano del lado afectado. Debido a lo anterior, disminuye la capacidad funcional de las personas de edad, por lo que requieren ayuda para la realización de las actividades de la vida diaria, como usar el baño, comer, bañarse, desplazarse por una habitación, comprar o preparar alimentos, ${ }^{4}$ convirtiéndose en una persona dependiente.

En los mayores de 65 años, se registra que el 14\% presentan dependencia leve (ayuda en menos de cinco actividades instrumentales), el 6\% dependencia moderada (ayuda en una o dos actividades básicas o más de cinco actividades instrumentales) y $12 \%$ dependencia grave (ayuda en tres o más actividades básicas de la vida diaria). ${ }^{5}$ El Consejo de Europa $^{5}$ define la dependencia como aquel estado en que se encuentran las personas que, por razones ligadas a la falta o a la pérdida de autonomía física, psíquica o intelectual, tienen necesidad de asistencia y/o ayudas importantes a fin de realizar los actos corrientes de la vida diaria. Una vez instalada la dependencia en el adulto mayor, es necesaria la presencia de una persona para la ayuda en las actividades de la vida diaria, es decir, un cuidador familiar. Según Pinto, el cuidador familiar es la persona que tiene un vínculo de parentesco o cercanía y asume el rol y la responsabilidad del cuidado de un ser querido que vive con enfermedad crónica. Este cuidador participa en la toma de decisiones, supervisa y apoya la realización de las actividades de la vida diaria para compensar la disfunción existente en la persona con enfermedad. ${ }^{6}$ Morris, Arias y Villaseca agregan que es la persona que sin compensación económica, brinda cuidado al adulto mayor que no puede hacerlo por sí mismo. ${ }^{7} \mathrm{El}$ cuidador familiar de un adulto mayor dependiente, en el desempeño de su tarea de cuidar, realiza una serie de actividades, experimenta una serie de vivencias y se enfrenta a diferentes situaciones problemáticas durante las 24 horas de cada día, por las cuales se ve transformada de forma negativa o positiva su vida cotidiana. Según La Parra, el $88 \%$ del cuidado para adultos mayores dependientes lo proporciona el cuidador familiar. ${ }^{8}$ Giraldo y Franco encontraron que el $48.7 \%$ de las cuidadoras invierten más de 16 horas al día en el cuidado. ${ }^{9}$ Una de las dimensiones afectadas es el espacio; Vida ${ }^{10}$ refiere que el cuidado de una persona en casa implica reorganizar y compartir el espacio entre el cuidador y adulto mayor, así como realizar adaptaciones físicas en la vivienda con la finalidad tanto de minimizar las dificultades del adulto mayor como para dar condiciones al cuidador familiar para proporcionar ayuda adecuada.

Creutzerbg refiere que entre los cuidados identificados que realiza el cuidador familiar en el día a día, está el apoyo en las actividades de cuidado personal y actividades domésticas. ${ }^{11}$ Camarano identificó que uno de los aspectos principales que afectan la vida cotidiana de la mayoría de los cuidadores familiares es la dificultad económica. ${ }^{12}$ Otras dificultades que se enfrentan en el cotidiano del cuidador familiar están relacionadas con la falta de conocimientos para realizar el cuidado, ${ }^{11}$ por lo que se presentan sentimientos de miedo a que empeore la salud del anciano, así como de impotencia al no poder ayudar a mejorar la capacidad funcional del adulto mayor. ${ }^{13}$ Vidal refiere que el cuidador familiar pasa por un gran desgaste físico y emocional, ${ }^{10}$ ya que entre más se involucra el cuidado en la vida del cuidador, más la cambia, y éste se percibe como una persona sin identidad, gustos y deseos, ya que el cuidar muchas veces significa anularse y dejar su vida de lado para asumir la vida del otro; ${ }^{14,15}$ dicha situación puede llevar a la aparición de enfermedades. ${ }^{16,17}$ Estos cuidadores utilizan dos veces más los servicios de salud mental que las personas que no realizan cuidados a la salud en el hogar. ${ }^{18}$

Para el abordaje teórico del cotidiano del cuidador familiar se emplea la teoría de Henry Lefebvre, quien define "lo cotidiano" como lo que no lleva fecha, lo insignificante 
aparentemente pero que da sentido, los hechos y fragmentos de la realidad que acontecen durante las 24 horas, como la síntesis de lo vivido. Se puede conocer a través de los componentes de la vida cotidiana como el espacio, el tiempo, las pluralidades de sentido, lo simbólico y las prácticas sociales. ${ }^{19}$ El espacio, subjetivamente, es el entorno de un individuo y de un grupo, es el horizonte dentro del cual se sitúan y viven los individuos; objetivamente, se relaciona con lo durable. El tiempo es el tiempo del cambio, y el cotidiano se compone de repeticiones: gestos en el trabajo y fuera del trabajo, movimientos mecánicos por horas, días, semanas, meses, años. Las pluralidades de sentido son los diferentes puntos de vista que se pueden condensar en ciertos elementos espaciales. Los actos, dramas individuales o colectivos son símbolos que parecieran ser trivialidades reiterativas, pero son más que eso: son aspectos de la vida cotidiana que el lenguaje no dice, es lo que evita decir, lo que no puede ni debe decir, como son los deseos; éstos se dan a conocer obscuramente atrás de los símbolos. Las prácticas sociales son la praxis repetitiva y praxis inventiva o creativa; ${ }^{19}$ son todos aquellos cuidados que realiza el cuidador familiar. Desde esta perspectiva, se planteó la pregunta de investigación: ¿cómo es el cotidiano del cuidador familiar del adulto mayor con dependencia?

Los objetivos planteados fueron describir, analizar e interpretar el cotidiano del cuidador familiar del adulto mayor con dependencia.

\section{METODOLOGÍA}

La presente investigación es de abordaje cualitativo descriptivo-exploratoria. De acuerdo con Minayo, ${ }^{20}$ el abordaje cualitativo responde a cuestiones particulares trabajando con universo de significados, motivos, aspiraciones, creencias, valores y actitudes que responden a un espacio más profundo de las relaciones, de los procesos y de los fenómenos involucrados. Estuvo constituida por ocho cuidadoras familiares de adultos mayores. Todos los adultos mayores presentaron dependencia total para las actividades básicas de la vida diaria, según la escala de Barthel. El cuidador familiar como mínimo tenía seis meses de desempeñar el rol de cuidador y, además, no recibía ningún tipo de remuneración económica a cambio del cuido del adulto mayor. Sus edades durante el cuidado oscilaron entre 50 y 60 años de edad. La selección de la muestra fue por conveniencia. Todas ellas cuidaban a su madre, padre o suegra cuando enfermaron y fueron dependientes. Se solicitó su participación voluntaria utilizando el consentimiento informado. La recolección de datos se realizó entre los meses de octubre del 2012 y mayo del 2013 en el domicilio del cuidador familiar del adulto mayor con dependencia, derechohabiente del Hospital "Lic. Adolfo
López Mateos", específicamente del Servicio de Extensión Hospitalaria. Los datos se recolectaron utilizando la técnica de la entrevista a través de la guía semiestructurada elaborada por la investigadora. La duración aproximada de cada entrevista fue de dos horas, utilizando una o dos sesiones. Las entrevistas se realizaron por el investigador, fueron grabadas y posteriormente transcritas para su análisis. Se llevó una bitácora, en la cual se recogieron notas de campo como comentarios y observaciones relacionadas con el entorno en ese momento, así como las reacciones a determinados temas a lo largo de la entrevista. Al final de la entrevista, también se anotaron las impresiones generales sobre el transcurso de la misma y sobre el informante. Se optó por el análisis temático según Minayo; ${ }^{21}$ se inició con leer y releer repetidas veces las transcripciones, buscando detectar los temas; posteriormente, se asignaron etiquetas con una palabra o frase a los fragmentos de texto que éstos representaban. Luego, se realizó una lectura fluctuante de cada entrevista anotando las primeras impresiones, buscando la coherencia interna de las informaciones a través de frases, palabras, adjetivos, concatenación de ideas y sentido general del texto para construir las categorías empíricas. Una vez codificados cada uno de los textos, se buscó integrar la información relacionada con cada categoría para reducirla a puntos esenciales. Posteriormente, se realizó la lectura transversal, para lo que se hizo el recorte de cada entrevista por temas y se agruparon las partes por semejanza, buscando percibir las conexiones entre ellas y guardándolas por categorías en carpetas, buscando comprender e interpretar aquello que fue expuesto como más relevante y representativo por el grupo estudiado. En cada paso, se buscó el significado de los pensamientos, sentimientos y comportamientos descritos en los textos; es decir, se interpretaron los datos. Por último, se realizó una interpretación global de los resultados de la investigación, explicando cómo al relacionarse las áreas temáticas se conoce el cotidiano del cuidador familiar del adulto mayor con dependencia. Para el rigor científico, se consideraron los criterios de credibilidad, la transferencia, la consistencia y la confirmación.

\section{RESULTADOS Y DISCUSIÓN}

Participaron en el estudio ocho mujeres cuidadoras familiares, de entre 50 y 60 años de edad. De las ocho, seis son hijas, una esposa y una nuera. En cuanto al estado civil, tres son divorciadas, una viuda y cuatro casadas. Sobre su condición laboral, dos tienen negocio propio, cuatro son amas de casa y dos son jubiladas. El nivel socioeconómico de seis es medio-bajo y de dos, medio-medio. Todos los adultos mayores presentaron dependencia total para las actividades básicas de la vida diaria según la escala de 
Barthel. La cuidadora familiar, como mínimo, tenía seis meses de desempeñar el rol de cuidador y, además, no recibía ningún tipo de remuneración económica a cambio del cuido del adulto mayor.

Analizar los discursos obtenidos de las cuidadores familiares de adultos mayores con dependencia que participaron en la investigación nos permitió conocer el cotidiano de dicho actor social, y emergieron dos categorías empíricas: "tiempo y espacio síntesis de lo vívido en el cotidiano", con las subcategorías "reconfigurando el espacio", "espacio y tiempo de aprendizaje", y "cambió mi vida en el tiempo y espacio"; y la segunda categoría, "simbolizando el cuidado en el cotidiano", con las subcategorías "ser cuidadora es ser mujer", "máscaras del cuidado" y "pluralidad de sentidos". El presente artículo se centra en describir la primera subcategoría de la primera categoría: "reconfigurando el espacio".

\section{Tiempo y espacio síntesis de lo vivido en el cotidiano}

Lo cotidiano se observa en la trama de las 24 horas de un día cualquiera y de una persona cualquiera. ${ }^{20}$ La vida cotidiana nos rodea y nos acerca; en el mismo tiempo y en el mismo espacio, está en nosotros y nosotros en ella. ${ }^{21}$ El espacio es, por lo tanto, un medio y un entorno que no tiene "existencia en sí", sino que remite al tiempo existencial y simultáneamente esencial. También, el espacio es la manifestación de un empleo de tiempo en una determinada sociedad, por lo que prevalecen su ideología y su concepción de las relaciones sociales. ${ }^{22}$ Entonces, el espacio existe en función del tiempo, y en éste se manifiesta la ideología y las relaciones sociales prevalentes de las personas, así como la apropiación de los lugares específicos al cargarlos de sentido y significado en el tiempo vívido.

En este sentido, el tiempo y espacio vividos del cuidador familiar son el entorno donde dice y hace su vida durante las 24 horas de cada día al cuidado del adulto mayor dependiente. También es llamado "espacio de vida". ${ }^{22}$ En esta categoría emergió la subcategoría "reconfigurando el espacio".

\section{Reconfigurando el espacio}

La casa es el espacio objetivamente, ${ }^{23}$ el lugar en donde el cuidador familiar brinda cuidados para satisfacer las necesidades del adulto mayor dependiente. Y ante la responsabilidad cotidiana de cuidar, el cuidador acomoda, compone y organiza los muebles, áreas físicas, etcétera, que se encuentran en su espacio, es decir, reconfigura el espacio físico para estar al pendiente de lo que necesita el adulto mayor y brindar un cuidado seguro; además, para facilitar su labor de cuidador. Entre los cambios que hace en el espacio físico son los que realiza para estar más cerca del adulto mayor dependiente para vigilarlo durante la noche, como se evidencia en los siguientes discursos:

Yo me quedo en el mismo cuarto que mi mamá, para estarla vigilando, pues luego tiene muchas flemas (CAR-4).

Yo me quedo aquí en un catre; sí, aquí en la sala, para estar al pendiente de lo que necesite mi suegra en la noche (ROS-8).

Le dije a mi hijo: "Tengo que cambiarte de recámara para estar más cerca de tu papá, porque si me habla, no lo voy a escuchar". Yo no me duermo en la misma cama, pues es una cama de hospital, me duermo en la sala porque así escucho mejor, me da como más seguridad y tranquilidad (MAC-6).

Esta recámara era de mi hijo... pero se la dejamos a la abuelita porque es muy latosa, de repente en la noche se despierta y está platicando o grita; entonces, si está cerca de mis hijos, no los deja dormir (MON-2).

Según Ferrer, en el cuidador emerge una preocupación permanente que muchas veces obliga a estar pendiente todo el tiempo de los movimientos o comportamiento del adulto mayor, por lo que el cuidador adopta una actitud vigilante. ${ }^{23}$ En este sentido, Vidal refiere que reorganizar el espacio es necesario con la finalidad de minimizar las dificultades del adulto mayor, así como para dar condiciones al cuidador de proporcionar la ayuda adecuada las 24 horas del día. ${ }^{10}$ Por lo tanto, el cuidado de una persona en casa implica reorganizar y compartir el espacio entre el cuidador y adulto mayor. ${ }^{10,24}$ De la Cuesta refiere que los espacios habituales de la casa se reconfiguran; el cuidador cambia su espacio para dormir con la finalidad de estar más cerca, se duerme en la misma cama que el adulto mayor dependiente para oírlo si necesita, y así facilitar la vigilancia y el cuidado. ${ }^{25}$ Según Esandi, cuando ocurre una caída o un accidente, el cuidador aprende a identificar las barreras que representan un obstáculo para el enfermo, a conocer los factores y estrategias que le van a ayudar a proporcionar un cuidado seguro, por lo que realiza cambios en el entorno para prevenir un nuevo accidente. ${ }^{26}$ Los resultados de la presente investigación coinciden con lo reportado en las investigaciones antes referidas, ya que en los discursos se refleja cómo una vez que el cuidador asume el cuidado del adulto mayor dependiente, tiene que hacer modificaciones en su espacio cotidiano, es decir, reconfigurarlo en función de las necesidades de cuidados del adulto mayor dependiente para compensar las discapacidades. El cuidador se cambia a un lugar más cercano o se muda a la cama del adulto mayor durante la noche para estar más cerca y vigilarlo, lo que le proporciona tranquilidad y seguridad. Muchos de estos cambios en el espacio del cuidador los realiza cuando el adulto mayor presenta deterioro cogni- 
tivo, el adulto mayor durante la noche presenta conductas que colocan en riesgo su integridad. También los espacios se reconfiguran cuando el adulto mayor durante la noche requiere que el cuidador realice determinados procedimientos, como es la aspiración de secreciones y la colocación de posición semi-fowler para evitar la broncoaspiración ante la presencia de enfermedades respiratorias.

El cuidador reconfigura sus espacios a manera de adaptarlos para el trabajo terapéutico; sin embargo, también se reconfiguran en función de las incomodidades que causa la conducta del adulto mayor al resto de la familia, resultados que coinciden con De la Cuesta, que refiere que el cuidador modifica su espacio considerando el bienestar de todos los integrantes que habitan la casa; es decir, también crea un espacio habitable para la familia. ${ }^{26} \mathrm{~A}$ partir de las prácticas que genera el cuidador familiar define que el espacio es suyo y sólo suyo, es decir, diferente de otros espacios generados por otras personas. Es así como el cuidador moldea su espacio y a la vez es moldeado por él. ${ }^{20} \mathrm{Su}$ espacio privado se convierte en espacio público en aras de satisfacer las demandas de cuidado del adulto mayor dependiente, dejando a un lado su vida propia.

A la reconfiguración del espacio físico de la casa se suma la introducción de objetos a ella. Al espacio cotidiano del cuidador se integra la presencia de equipo médico y ayudas técnicas para el cuidado, como silla de ruedas, cama de hospital, WC portátil; su espacio objetivo, o lo que constituye la casa, se vuelve un espacio de hospital, como se evidencia en los discursos:

Cuando mi esposo se puso mal y ya no pudo moverse, tuvimos que colocar una cama de hospital. (MAC-6)

Antes de traer a mi mamá, yo le adapte el espacio... tengo un aspirador porque ella tuvo un proceso respiratorio y un nebulizador, tengo sus medicamentos; ése es su clóset donde tengo sus cosas. (GLO-7)

Me dice "es que me anda del baño", entonces ni modo, trajimos el baño portátil. (Ma-1)

Según Lefebvre, el espacio cotidiano de toda persona está poblado de signos, como objetos funcionales producto de la actividad práctica que se realiza. ${ }^{22}$ Esto se refleja en el espacio del cuidador familiar del adulto mayor dependiente, ya que se rodea de ayudas técnicas -como baño portátil-o equipo médico - como camas de hospital, aspiradores, nebulizadores, etcétera-, de los cuales se vale para compensar las discapacidades del adulto mayor dependiente. Estas ayudas técnicas ahora constituyen el habitar, envuelven y significan las relaciones sociale ${ }^{22}$ del cuidador familiar. Esto coincide con De la Cuesta, que refiere que con el tiempo y a medida que la enfermedad progresa, los cuidadores familiares tienen que rodearse de instrumentos que faciliten el cuidado cada vez más dependiente y complejo. ${ }^{26}$ De esta manera, el mundo material del cuidador se va llenando de implementos ajenos a la cotidianidad hasta antes de asumir el rol de cuidador y que ahora pasan a formar parte de la reconfiguración de su espacio cotidiano. Todos estos objetos que compraron o pidieron prestados pasan a ser un elemento visible y central en el espacio del cuidador.

\section{CONCLUSIONES}

A medida que la enfermedad avanza y el adulto mayor se torna con grados de dependencia mayor, los cuidadores cambian y adaptan la vivienda para brindar los cuidados seguros y con la mayor calidad posible, así como para que el cuidador se sienta más seguro y tranquilo, sobre todo cuando el adulto mayor presenta deterioro cognitivo. El espacio del cuidador familiar de un adulto mayor dependiente es característico de los cuidados que realiza; está constituido de todo aquello que le sirve para brindar cuidados en las mejores condiciones posibles, tanto para el cuidador familiar como para el adulto mayor. Sin embargo, para el cuidador pierde el significado de espacio privado, pues se convierte en un espacio hospitalario y denota tiempo para cuidar las 24 horas de cada día.

\section{BIBLIOGRAFÍA}

1. Instituto Nacional de Estadística y Geografía. INEGI. México en cifras. Información nacional por entidad federativa y municipios [Internet]. México: INEGI; 2010 [acceso 19 de marzo del 2012]. Disponible en: http://bit.ly/rVqpew

2. Secretaría de Salud. SSA. Programa Nacional de Salud 2007-2012 [Internet]. México: SSA; 2007 [acceso 20 de marzo del 2012]. Disponible en: http://bit.ly/19lGTV8

3. Cardona AD, Estrada RA, Agudelo GHB. Envejecer nos toca a todos. Caracterización de algunos componentes de calidad de vida y de condiciones de salud de la población adulta mayor [Internet]. Medellín: Universidad de Antioquía, Facultad Nacional de Salud Pública; 2002 [acceso 9 de septiembre de 2012]. Disponible en: http://bit.ly/18idMIT

4. Instituto Nacional de Estadística Geografía e Informática. INEGI. Los adultos mayores en México. Perfil sociodemográfico al inicio del siglo XXI [Internet]. México: INEGI; 2005 [acceso de 17 agosto de 2012]. Disponible en: http://bit.ly/12imhbs

5. Sociedad Española de Geriatría y Gerontología, SEGG. Tratado de geriatría para residentes. Madrid: Sociedad Española de Geriatría y Gerontología; 2006.

6. Pinto AN. Cuidar en el hogar a personas con enfermedad crónica, su familia y cuidador. Colombia: Universidad de Colombia, Facultad de Enfermería; 2010.

7. Morris R, Arias A, Villaseca M. Necesidades y demandas de los cuidadores primarios de pacientes terminales de cáncer en atención primaria: artículos [Internet]. 1995 [acceso 10 de noviembre de 2003]. Disponible en: http://geocities.com/hotspring/spa/7712/indice.html

8. La Parra D. Contribución de las mujeres y los hogares más pobres a la producción de cuidados de salud informales. Gaceta Sanitaria. 2001; 15 (6): 498-505.

9. Giraldo MCL, Franco AGM. Calidad de vida de los cuidadores familiares. Revista Aquichan. 2006; 6 (1): 38. 
10. Vidal ECJ. O perfil do cuidador familiar do paciente com séquela de accidente vascular encefálico [Dissertação de Mestre em Familia na Sociedade Contemporánea]: Universidad de Católica Do Salvador [Internet]. 2005 [acceso 25 agosto 2012]. Disponible en: http://bit.ly/17SkNJk

11. Creutzerbg M. Vivencias de familias de clase popular cuidadoras de pessoas idosa fragilizada: subsídios para o cuidado de enfermagem domiciliar [Dissertação de Maestre en Enfermagem]: Univeridade Federal Do Rio Grande Do Sul Porto Alegre, Janeiro [Internet]. 2000 [acceso 25 octubre 2012]. Disponible en: http://bit.ly/17SkNJk

12. Camarano AA. Os novos idosos brasileiros muito além dos 60? IPEA Instituto de Pesquisa Económica Aplicada [Internet]. 2004 [acceso 8 septiembre 2012]. Disponible en: http://bit.ly/15fv3xC

13. Jandrey CV. O cuidador familiar de pessoa idosa: o desafio de cuidar de quem cuida. [Dissertação de Mestrado em Teologia]: Faculdades EST. Programa de Pós-Graduação Teología Práctica. Sao Leopoldo [Internet]. 2009 [acceso 30 agosto 2012]. Disponible en: http://bit.ly/17SkNJk

14. Fernández MGM, García TR. Estrutura conceitual da tensão do cuidador familiar de idosos dependentes. Rev Eletr Enf [Internet]. 2009 [acceso 3 agosto de 2013]; 11 (3): 469-476. Disponible en: http://bit.ly/ldz8V11

15. Úbea BI. Calidad de vida de los cuidadores familiares: evaluación mediante un cuestionario. [Disertación Doctoral]: Escuela de Enfermería de la Universidad de Barcelona. 2009.

16. Rodrigues SLA, Watanabe HAW, Derntl AM. A saúde de idosos que cuidam de idosos. Rev Esc Enferm USP [Internet]. 2006 [acceso 2 agosto 2012]; 40 (4): 493-500. Disponible en: http://bit.ly/1fnHN73
17. Schossler T, Crossetti MG. Cuidador domiciliar do idoso e o cuidado de si: uma análise a través da teoría do cuidado humano de Jean Watson. Texto Contexto Enferm. 2008; 17 (2): 280-287.

18. Amstrong P, Kits O. One hundred years of caregiving. En: Nigenda G, Matarazzo C, López OM. Los cuidados a la salud en el hogar: tendencias e inequidades. Análisis desde una perspectiva de género. México: Secretaría de Salud. Fundación Mexicana para la Salud; 2005.

19. Lefebvre $\mathrm{H}$. La vida cotidiana en el mundo moderno. $3 \mathrm{a}$ ed. Madrid: Alianza Editorial; 1984.

20. Minayo MC. La artesanía de la investigación cualitativa. Buenos Aires: Lugar Editorial; 2009

21. Lefebvre H. De lo rural a lo urbano. 4a ed. Barcelona: Editorial Península; 1978.

22. Lefebvre H. Critique de la vie quotidien. Vol. II. Fondements d'une sociologie de la quotidianneté. Perís: L’Arche Editeur; 1961.

23. Ferrer HME. El aprendizaje de cuidados familiares a pacientes con Alzheimer. El ingenio desarrollado sobre la marcha en una compleja realidad. [Disertación Doctoral]: Universidad de Alicante; 2008.

24. Buil P, Diez EJ. Anciano y familia. Una relación en evolución. ANALES Sis Navarra, 22 (Supl.I):19-25.

25. De la Cuesta BC. Cuidado artesanal: la intervención ante la adversidad. Antioquía, Colombia: Universidad de Antioquía, Facultad de la Universidad de Antioquía; 2004.

26. Esandi LN, Canga AA. Familia cuidadora y enfermedad de Alzheimer: una revisión bibliográfica. Gerokomos. 2011; 22 (2): 56-61. 